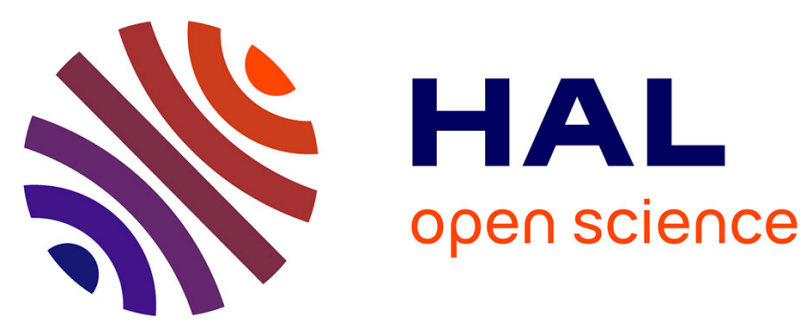

\title{
Modélisation numérique du flambage des plaques minces et applications au laminage
}

\author{
Sami Abdelkhalek, Hamid Zahrouni, Michel Potier-Ferry, Pierre \\ Montmitonnet, Nicolas Legrand, Pascal Buessler
}

\section{- To cite this version:}

Sami Abdelkhalek, Hamid Zahrouni, Michel Potier-Ferry, Pierre Montmitonnet, Nicolas Legrand, et al.. Modélisation numérique du flambage des plaques minces et applications au laminage. CFM'09 XIXème Congrès Français de mécanique, Aug 2009, Marseille, France. 6 p. hal-00509386

\section{HAL Id: hal-00509386}

https://hal-mines-paristech.archives-ouvertes.fr/hal-00509386

Submitted on 26 Jan 2012

HAL is a multi-disciplinary open access archive for the deposit and dissemination of scientific research documents, whether they are published or not. The documents may come from teaching and research institutions in France or abroad, or from public or private research centers.
L'archive ouverte pluridisciplinaire HAL, est destinée au dépôt et à la diffusion de documents scientifiques de niveau recherche, publiés ou non, émanant des établissements d'enseignement et de recherche français ou étrangers, des laboratoires publics ou privés. 


\title{
Modélisation numérique du flambage des plaques minces et applications au laminage
}

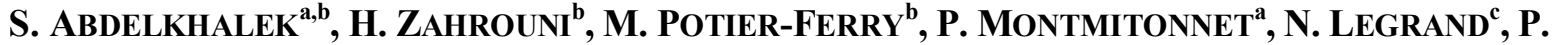 \\ BUESSLER $^{\mathrm{c}}$ \\ a. Ecole des Mines de Paris-ParisTech-CEMEF (UMR CNRS 7635), BP-207 -1, rue Claude Daunesse 06904 \\ Sophia-Antipolis-Cedex-France.
}

b. LPMM (UMR CNRS 7554) - ISGMP - Université de Paul Verlaine-Metz, Ile de Saulcy 57045 Metz -Cedex France.

c. ArcelorMittal Research Maizières, R\&D Industrial Operations - BP 30320 - Voie Romaine 57283 Maizières Les Metz-Cedex-France.

\section{Résumé :}

Dans certains procédés de mise en forme, tel que le laminage, l'apparition des contraintes résiduelles est inévitable et peut engendrer le flambage qui se traduit en défauts géométriques. Ceci exige la modélisation de ce phénomène d'instabilité mécanique afin de bien définir la forme et l'amplitude de ces défauts. Cela nécessite un modèle complet ayant des capacités pour le calcul du post-flambage. Dans le présent article, on présente un modèle de flambage possédant la faculté de détecter la charge critique du flambage et la forme post-flambée d'une plaque sous contraintes résiduelles. Des applications à des cas académiques et un cas de laminage seront aussi traités.

\begin{abstract}
:
In some material forming processes, as in strip rolling, residual stresses inevitably appear and may result in buckling which generates geometric defects. This requires predicting this mechanical instability phenomenon in order to define defect geometry and amplitude. For that reason, a complete buckling model with a post-buckling capability is recommended. In this paper, we present a residual stress buckling plate model which has a critical load and post-buckling computing capability. Several academic applications and a rolling case are treated.
\end{abstract}

Mots clefs : Laminage, défauts de planéité, flambage, post-flambage, contraintes résiduelles.

\section{Introduction}

Dans une structure, la répartition des champs de contraintes résiduelles peut être plus ou moins variable et capable de causer le flambage quand ces contraintes dépassent un certain niveau critique. Les origines de ces contraintes résiduelles sont très variées et on peut citer :

- Les origines thermiques où un matériau est soumis à des variations de température sans avoir la possibilité de se dilater.

- Les origines mécaniques quand un matériau subit une déformation plastique hétérogène.

Dans la plupart des procédés de mise en forme, notamment en laminage des tôles minces, le flambage est incontrôlable et peut avoir des effets néfastes sur la structure. De ce fait, ce phénomène doit être analysé assez profondément afin de comprendre ses causes, pouvoir le modéliser et essayer de l'éviter.

En laminage, lors de l'écrasement de la tôle dans l'emprise, les cylindres de travail subissent une déformation élastique qui est généralement inévitable. Ainsi, la déformation plastique de la tôle à cet endroit n'est pas homogène sur toute la largeur de la table, ce qui engendre l'apparition des contraintes résiduelles d'origine 
mécanique. En conséquence, le flambage peut avoir lieu, donnant naissance à des ondulations de formes diverses que l'on nomme dans ce contexte « défauts de planéité ». Par ailleurs, un modèle éléments finis 3D basé sur la Méthode Asymptotique Numérique (MAN) et une formulation de coque [1] à été développé. Ce modèle doté d'une capacité pour simuler le flambage des coques, nous a permis de modéliser les défauts de planéité en laminage. D'autre part, il est particulièrement difficile de trouver dans la littérature des études de post-flambement et les auteurs [2]-[6] se limitent à des calculs de flambage linéaire, c'est-à-dire uniquement à la recherche des modes propres de flambage. Cette carence permettra d'avoir uniquement une idée sur la forme des défauts en laminage et non de les quantifier (en UI par exemple) ou d'observer le comportement de la structure après le flambage sous contraintes résiduelles; particulièrement dans certains cas où on a observé qu'une tôle peut se transformer d'un mode à un autre au cours du post-flambage.

Dans le présent article, on montrera des applications de flambage de plaques métalliques sous contraintes résiduelles à l'aide d'un modèle ayant des capacités à faire des calculs de post-flambage, en passant par des cas académiques simples à un cas d'application au laminage.

\section{Formulation et Méthode Asymptotique Numérique}

Les équations du problème sont équivalentes à la stationnarité de la fonctionnelle de Hu-Washizu qui s'écrit comme suit:

$$
\delta \varphi_{\mathrm{HW}}=\int_{v}\left\{{ }^{t} \delta S:\left[\left(\gamma_{u}+\tilde{\gamma}\right)-D^{-1}: S\right]+{ }^{t} S:\left[\delta \gamma_{u}+\delta \tilde{\gamma}\right]\right\} d v-\lambda P_{e}(\delta u)
$$

où $D$ est le tenseur des constantes élastiques, $S$ est le second tenseur de contraintes de Piola-Kirchoff, $\gamma_{u}$ est la déformation compatible de Green-Lagrange obtenue à partir du champ de déplacement $u$ qui est composé d'une partie linéaire et d'une partie quadratique : $\gamma_{u}=\gamma_{l}(u)+\gamma_{n l}(u, u) . \tilde{\gamma}$ est la déformation additionnelle indépendante du déplacement, qui est choisie orthogonale au champ des contraintes, permettant d'éviter le phénomène de verrouillage. $P_{e}(\delta u)$ est le travail virtuel des efforts extérieurs et $\lambda$ est un paramètre de chargement. Ce dernier atteint une valeur critique $\lambda_{c}$ quand le flambage a lieu.

L'équation (1) peut s'écrire sous forme d'un résidu $R$ comme suit :

$$
R(U, \lambda)=L(U)+Q(U, U)-\lambda F=0
$$

où $L($.$) et Q(. .$,$) sont respectivement un opérateur linéaire et quadratique, F$ est le vecteur des efforts extérieurs.

\subsection{MAN pour le suivi de courbe}

La MAN consiste à chercher la solution du problème non linéaire (1) sous forme d'un développement asymptotique au voisinage d'une solution initialement connue $\left(U_{0}, \lambda_{0}\right)$ selon un paramètre « $a »$ :

$$
\begin{aligned}
& U(a)=U_{0}+\sum_{i=1}^{n} a^{i} U_{i} ; \lambda(a)=\lambda_{0}+\sum_{i=1}^{n} a^{i} \lambda_{i} \\
& a=\left\langle u-u_{0}, u_{1}\right\rangle+\left(\lambda-\lambda_{0}\right) \lambda_{1}
\end{aligned}
$$

Ici, $U=(u, \tilde{\gamma}, S)$ et $\mathrm{n}$ est l'ordre de troncature des séries.

Si on substitue (3) dans (2), le problème se transforme en une séquence de problèmes linéaires comme on le montre ci-dessous :

ordre 1 :

$$
\begin{aligned}
& L_{t}^{0}\left(U_{1}\right)=\lambda_{1} F \\
& \left\langle u_{1}, u_{1}\right\rangle+\lambda_{1}^{2}=1
\end{aligned}
$$

ordre $p(1<p \leq n)$ : 


$$
\begin{aligned}
& L_{t}^{0}\left(U_{p}\right)=\lambda_{p} F-\sum_{r=1}^{p-1} Q\left(U_{r}, U_{p-r}\right) \\
& \left\langle u_{p}, u_{1}\right\rangle+\lambda_{p} \lambda_{1}=0
\end{aligned}
$$

$L_{t}^{0}($.$) est l'opérateur tangent qui ne dépend que de la solution initiale.$

\subsection{Indicateur de bifurcation}

L'indicateur de bifurcation [7] [8] est obtenu en introduisant une perturbation fictive dans le problème. Il ne s'agit pas de modifier le problème initial mais de construire un problème auxiliaire qui permet d'évaluer l'indicateur sur toute la branche d'équilibre et de déterminer tous les points singuliers et leurs modes correspondants.

Soit $\Delta \mu . f$ une force de perturbation fictive appliquée à la structure dans un état déformé $(U, \lambda)$. $\Delta \mu$ représente l'intensité de cette force et $\Delta U$ la réponse à cette perturbation. Si on considère le problème (2) et en négligeant les termes quadratiques en $\Delta U$ à l'équilibre, la forme perturbée est décrite par l'équation suivante :

$$
L_{t}(\Delta U)=\Delta \mu f
$$

où $L_{t}()=.L()+.2 Q(U,$.$) est l'opérateur tangent défini au point d'équilibre considéré (U, \lambda)$.

Pour résoudre ce problème, on se donne une condition supplémentaire définie comme suit :

$$
\left\langle L_{t}^{0}\left(\Delta U-\Delta U_{0}\right), \Delta U_{0}\right\rangle=0
$$

où $L_{t}^{0}$ est l'opérateur tangent au point de départ $\left(U_{0}, \lambda_{0}\right)$ et $\Delta U_{0}$ est solution de $L_{t}^{0}\left(\Delta U_{0}\right)=f$.

L'indicateur de bifurcation $\Delta \mu$ peut être alors calculé à partir de (6) et (7):

$$
\Delta \mu=\frac{\left\langle\Delta U_{0}, f\right\rangle}{\left\langle L_{t}^{-1}(f), f\right\rangle}
$$

qui s'annule exactement aux points critiques.

Le problème ((6) et (7)) est résolu en utilisant la méthode asymptotique numérique. Les variables $\Delta U$ et $\Delta \mu$ sont recherchées sous la forme suivante :

$$
\Delta U(a)-\Delta U_{0}=\sum_{i=1}^{N} a^{i} \Delta U_{i} ; \Delta \mu(a)-\Delta \mu_{0}=\sum_{i=1}^{N} a^{i} \Delta \mu_{i}
$$

En injectant les équations (9) dans (6) et (7), on obtient :

à l'ordre 0 :

$$
L_{t}^{0}\left(\Delta U_{0}\right)=\Delta \mu_{0} . f
$$

On résout ce système en considérant $\Delta \mu_{0}=1$.

A l'ordre $p>1$ :

$$
L_{t}^{0}\left(\Delta U_{p}\right)=\Delta \mu_{p} f-2 \sum_{j=1}^{p} Q\left(U_{j}, \Delta U_{p-j}\right)
$$




$$
\left\langle\Delta U_{p}, f\right\rangle=0
$$

Les vecteurs $U_{i}$ et l'opérateur tangent $L_{t}^{0}$ sont ceux déterminés pour le calcul de la branche d'équilibre. Nous n'inversons pas de matrice supplémentaire dans cette procédure. Seuls des seconds membres sont calculés.

\section{Applications}

Le calcul de flambage par le modèle qu'on présente est réalisé en quatre étapes. Dans une première étape on applique à la tôle la contrainte de traction, ce qui nous permet d'approcher des conditions réelles de laminage et qui donne à la tôle une certaine rigidité au flambage. Ensuite, les champs de contraintes résiduelles sont introduits en tant que chargement. A ce stade, le modèle de flambage donne à la fois la charge critique et le mode correspondant. Dans une troisième étape, le modèle effectue un calcul en non linéarité géométrique. En effet, le mode de flambage est introduit comme un défaut dans la géométrie initiale de la structure et un suivi de courbe nous permet de connaître l'état post-flambé de la bande. Enfin, on peut avoir l'état de la tôle après le déchargement de la traction appliquée à la première étape. Les deux dernières étapes constituent une des particularités de ce modèle par rapport à ceux existants dans la littérature. Ainsi, il permet un calcul de post flambement connaissant la forme et l'amplitude des défauts de planéité en laminage qui pourront s'exprimer en UI.

\subsection{Plaque en appuis simples et sous contraintes résiduelles homogènes}

Soit une plaque de longueur $a=140 \mathrm{~mm}$, de largeur $b=100 \mathrm{~mm}$ et d'épaisseur $h=1 \mathrm{~mm}$. Elle est constituée d'un matériau dont les constantes d'élasticité sont données par le module de Young $E=200 \mathrm{GPa}$ et le coefficient de Poisson $v=0.3$. Cette plaque est en appuis simples sur les quatre bords et sous contraintes résiduelles homogènes, dont seulement la composante longitudinale est non nulle $\left(\sigma_{x x}=-\left(\frac{\pi h}{b}\right)^{2} \frac{E}{12\left(1-v^{2}\right)}\right)$. On supposera les conditions de symétrie par rapport au plan $(x, z)$ pour ne modéliser que la moitié de la structure (pour ce cas, on n'applique pas de traction à la plaque).

La figure 1 montre le mode post-flambé de la plaque ainsi que l'évolution du paramètre du chargement $\lambda$ en fonction du déplacement hors plan d'un nœud considéré (nœud 81). En examinant cette évolution, on observe la bifurcation qui se produit quand $\lambda$ s'approche de $\lambda_{c}$ (ici $\lambda_{c}=4.46$ qui correspond à la valeur analytiquement obtenue par [9] $\sigma_{c}=4.59 \mathrm{MPa}$ ) et se sature plus ou moins malgré que la structure garde une faible rigidité de flexion durant le flambage (adoucissement de la pente de la courbe $\lambda=f\left(u_{z}\right)$ ).

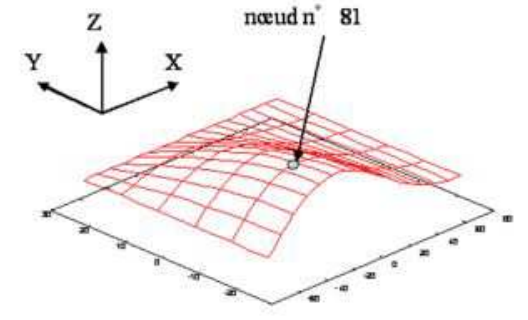

(a)

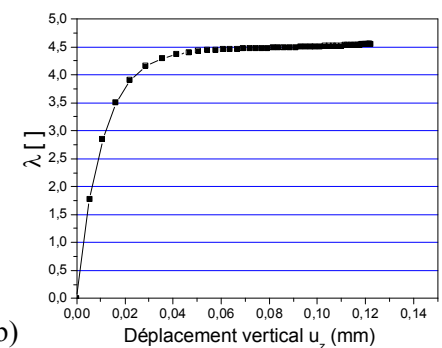

(b)

FIG 1 - (a) : Etat post-flambé d'une plaque en appuis simples sur les quatre bords, soumise à une contrainte résiduelle homogène $\sigma_{x x}$. (b) : Evolution du paramètre du chargement $\lambda$ au cours du flambage.

\subsection{Plaque sous contraintes résiduelles longitudinales hétérogènes dans la largeur}

Cette fois on considère un cas qui a été traité dans [4] qui constitue une plaque dont les propriétés élastiques sont identiques au cas précédent mais de longueur $a=500 \mathrm{~mm}$, de largeur $b=100 \mathrm{~mm}$ et d'épaisseur $h=1 \mathrm{~mm}$. La plaque est soumise à un profil de contraintes longitudinales résiduelles $\sigma_{x x}(y)$ comme montré dans la figure 2-a. Par ailleurs, pour obtenir des bords longs, les auteurs fixent le centre de la plaque et pour obtenir un centre long, ils fixent les bords. Ces conditions supplémentaires au problème leur permettent d'aboutir aux résultats souhaités. Ainsi, pour ce profil typiquement obtenu en laminage, [4] fixent le centre de la plaque en supposant 
qu'a priori le mode de flambage est en bords longs (ou bords ondulés). En revanche, dans le modèle qu'on présente, aucune de ces conditions supplémentaire n'est imposée afin d'obtenir ce type de résultats. Toutefois, dans le but de faire une comparaison avec les travaux de [4], on adoptera les mêmes conditions : une fixation du centre de la plaque ayant les bords libres et les deux extrémités en appuis simples. Les figures 2-b et c montrent que les résultats sont quasi identiques sauf que notre modèle est capable de fournir la forme post-flambé avec les amplitudes des défauts qui sont de l'ordre de $2 \mathrm{~mm}$.

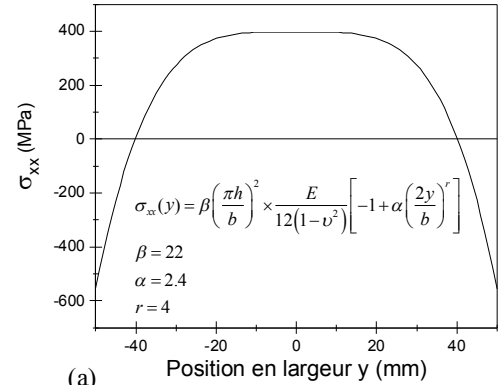

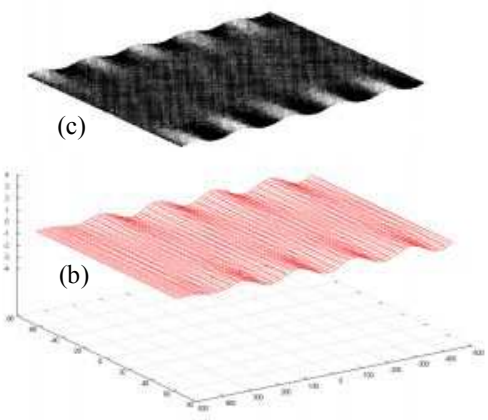

FIG 2 - Comparaison entre les résultats obtenus par [4] (a) et ceux qui sont obtenus par le présent modèle de flambage (b) pour les conditions citées dans le paragraphe 3.2.

\subsection{Application au laminage}

Pour un cas de laminage décrit dans le tableau 1, où on ne considère que la partie de la tôle située en aval de l'emprise tel que la condition de symétrie au centre a été adoptée. Dans le modèle de flambage, la sortie de l'emprise a été assimilée à un encastrement. A l'autre extrémité, on impose une condition d'appui simple et une contrainte de traction dans le sens horizontal, correspondant à la tension de bande pendant le laminage. Quant au bord (la rive de la bande), il a été considéré libre.

Dans une étude précédente [10], on a montré que le flambage qui se produit hors emprise n'a pas d'influence sur cette dernière. En se basant sur cette hypothèse, la modélisation des défauts de planéité en laminage est fondée sur une procédure découplée. Cette procédure consiste à utiliser ce modèle de flambage en chainage avec un modèle nommé Lam3/Tec3 basé sur la MEF 3D adapté à la simulation de laminage des produits longs, mais qui n'est pas capable de tenir compte du flambage sous contraintes résiduelles. Ainsi, un premier calcul de laminage est effectué à l'aide de Lam3/Tec3, sans tenir compte du flambage, fournissant des champs de contraintes résiduelles, y compris hors emprise. Ces champs de contraintes sont utilisés ensuite comme contraintes résiduelles pour le calcul de flambage qui permet d'obtenir la charge critique, le mode de flambage ainsi que l'état post-flambé avec la nouvelle (et réelle) répartition des contraintes dans la tôle. Toutefois, la différence entre la topologie du maillage de Lam3/Tec3 et celle du modèle de flambage fait appel à l'utilisation de la méthode des moindres carrés mobiles (MLS) afin d'effectuer le transfert des champs.

Pour ce cas traité, on montre sur les figures 3-a et b que des défauts sous forme de plis longitudinaux apparaissent dès la sortie de l'emprise. Ces défauts qui apparaissent généralement à cause de la traction, s'amplifient quand la traction est éliminée en passant de 1 à $5 \mathrm{~mm}$ environ. De plus, on note l'état flambé de la tôle, qui est non désirable, après sont relâchement.

\begin{tabular}{|l|l|l|l|l|}
\hline Largeur de la bande & Epaisseur d'entrée & Epaisseur de sortie & $\begin{array}{l}\text { Contrainte de } \\
\text { traction }\end{array}$ & $\begin{array}{l}\text { Propriétés élastiques (Module } \\
\text { d'Young et coefficient de Poisson ) }\end{array}$ \\
\hline $1232 \mathrm{~mm}$ & $0,409 \mathrm{~mm}$ & $0,279 \mathrm{~mm}$ & $58 \mathrm{MPa}$ & $E=210 \mathrm{GPa}, v=0,3$ \\
\hline
\end{tabular}

TAB 1 - Données du cas de laminage traité.

\section{Conclusion}

Dans cet article on a présenté un modèle de flambage sous contraintes résiduelles basé sur la méthode asymptotique numérique. Ce modèle a été utilisé dans le but de combler l'incapacité à simuler le flambage sous contraintes résiduelles (d'où les défauts de planéité) à l'aide de Lam3/Tec3, qui est un modèle éléments finis 3D dédié pour la modélisation du laminage. Les contraintes résiduelles sont alors extraites d'un calcul préalable de 
laminage par Lam3/Tec3 sans tenir compte du flambage. Ce dernier est ensuite calculé par le modèle considéré auquel nous lui transférons les contraintes résiduelles.

Pour simuler un cas avec une configuration proche des conditions de laminage, quatre étapes ont été adoptées : mise sous tension, recherche de modes, post-flambage et relâchement de la traction qui permet d'avoir une idée sur l'état d'une tôle laminée après le découpage. Les résultats obtenus sont satisfaisants et montrent des défauts de planéité qui sont rencontrés sur les lignes de laminage.
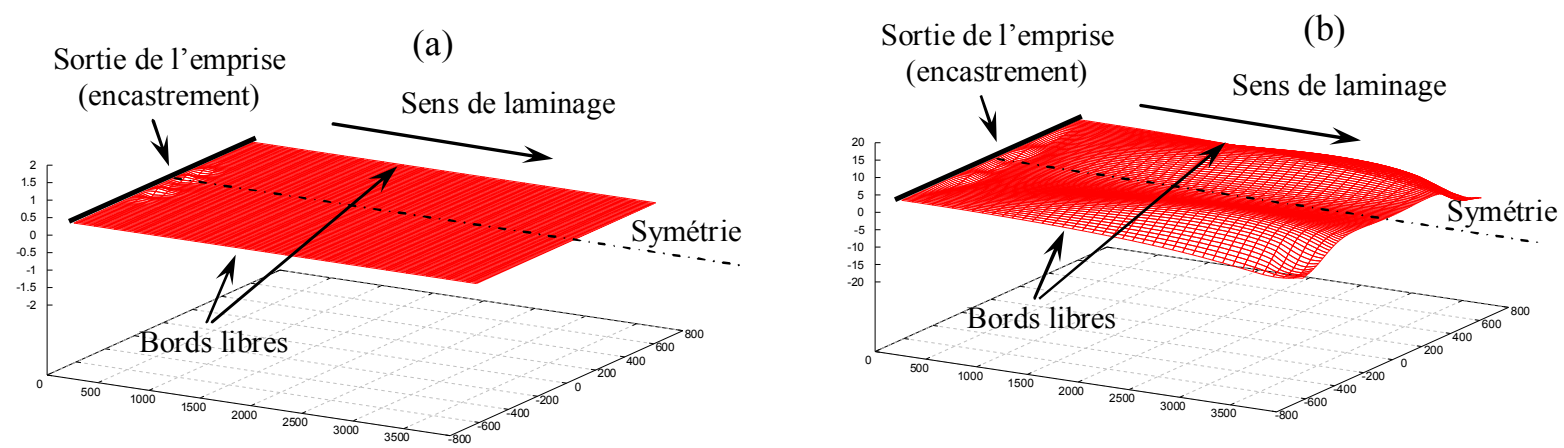

FIG 3 - Défauts de planéité quand la tôle est sous traction (a) et après sont relâchement (b).

\section{References}

[1] H. Zahrouni, B. Cochelin and M. Potier-Ferry. Computing finite rotations of shells by an asymptoticnumerical method. Computer Methods in Applied Mechanics and Engineering, 175, 71-85, 1999.

[2] Y. Tosawa. Analysis of three dimensional deformation in strip rolling taken deformation of rolls into consideration. Advanced technology of plasticity, 2, 1151-1160, 1984.

[3] C. D. Coman, A. P. Bassom. An asymptotic description of the elastic instability of twisted thin elastic plates. Acta Mechanica, 200, 59-68, 2008.

[4] A. Bush, R. Nicholls and J. Tunstall. Stress levels for elastic buckling of rolled strip and plate. Ironmaking and steelmaking, vol. 28, 481-484, 2001.

[5] F. G. Rammerstorfer, F. D. Fisher, N. Friedl. Buckling of free infinite strips under residual stress and global tension. Journal of applied mechanics, 68, 399-404, 2001.

[6] F. D. Fisher, F. G. Rammerstorfer, N. Friedl and W. Wisser. Buckling phenomena related to rolling and levelling of sheet metal. International journal of mechanical sciences, 42, 1887-1910, 2000.

[7] E.H. Boutyour, H. Zahrouni, M. Potier-Ferry and M. Boudi. Bifurcation Points and Bifurcated Branches by an Asymptotic Numerical Method and Padé Approximants. International Journal for Numerical Methods in Engineering, 60, 1987-2012, 2004.

[8] P. Vannucci, B. Cochelin, N. Damil, M. Potier-Ferry. An asymptotic numerical method to compute bifurcating branches. International Journal for Numerical Methods in Engineering, 4, 1365-1389, 1998.

[9] S. P. Timoshenko, J. M. Gere, Theory of elastic stability. Mc Graw Hill Book Company, Inc, New York, Second edition, 1961.

[10] S. Abdelkhalek, P. Monmitonnet, N. Legrand, P. Buessler. Manifested flatness predictions in thin strip cold rolling. In 11th ESAFORM conference forging and rolling, 2008. 